\title{
Composição Mineral de Ritmitos do Araripe por Análise Petrofísica
}

\author{
Coura, R.L.C. ${ }^{1}$; Soares, J.A. ${ }^{1}$; Gomes,I.F. ${ }^{2}$ \\ ${ }^{1}$ Universidade Federal de Campina Grande, ${ }^{2}$ Universidade Federal de Pernambuco
}

Copyright 2016, SBGf - Sociedade Brasileira de Geofísica

Este texto foi preparado para a apresentação no VII Simpósio Brasileiro de Geofísica. Ouro Preto, 25 a 27 de outubro de 2016. Seu conteúdo foi revisado pelo Comitê Técnico do VII SimBGf, mas não necessariamente representa a opinião da SBGf ou de seus associados. É proibida a reprodução total ou parcial deste material para propósitos comerciais sem prévia autorização da SBGf.

\section{Resumo}

In this paper petrophysical properties measured in rythmite rock samples from Araripe basin were used for mineral composition analysis. The petrophysical experiments done were grain and total density, porosity and elastodynamic properties. The elastic velocities showed an intermediate relationship between the Raymer and the Wyllie models. Contents of densest minerals control elastic velocities values. The dispersion observed in this relationship probably is caused by predominant porosity type. A low level of elastic anisotropy was observed. Through a biphasic model for mineral composition, three possible scenarios, which, further the occurrence of calcite, each one account for dolomite, pyrite or ankerite. Additional analyses need to be realized to decide which of them the most suitable scenario is.

\section{Introdução}

A Bacia do Araripe está localizada no Nordeste do Brasil, entre os lineamentos de Patos e Pernambuco. É uma extensa bacia interior dominada pela Chapada do Araripe e que também compreende a região do Vale do Cariri. Seus limites se estendem pelos estados do Ceará, Piauí, Pernambuco e Paraíba numa área de aproximadamente $10.000 \mathrm{~km}^{2}$.

O Andar Alagoas na Bacia do Araripe corresponde ao intervalo cronoestratigráfico que registra o estágio pósrifte da evolução cretácea das bacias marginais brasileiras (Assine, 1992). Esse intervalo estratigráfico é composto pelas formações Barbalha e Santana. A Formação Santana se subdivide nos membros Crato (inferior) e Membro Romualdo (superior). O Membro Crato é constituído por bancos de calcários laminados intercalados com folhelhos, siltitos e arenitos.

De acordo com Neumann et al. (2003), as principais características e distribuição da matéria orgânica tem sido estudadas em várias unidades lacustrinas AptianasAlbianas da Bacia do Araripe. Seis fácies principais foram distinguidas em termos de suas características litológicas - sedimentológicas e do tipo e quantidade de matéria orgânica: (1) laminitos e arenitos massivos a grosseiramente laminados, de ambientes lacustrinos deltaicos marginais, encontrados na forma de lentes e preenchimento de canais; (2) laminitos massivos a pobremente laminados característicos de ambiente lacustrino marginal oxidante; (3) laminitos de ambiente lacustre interior, oxidante a suboxidante, e laminitos bioclásticos laminados; (4) laminitos escuros típicos de ambiente lacustre interior redutor; (5) ritmitos carbonato argila escura típico de ambiente lacustre interior redutor; e (6) calcários micríticos escuros finamente laminados típicos de ambiente lacustre interior redutor.

Este trabalho trata da análise das propriedades petrofísicas da fácies (5): os ritmitos que ocorrem na Bacia do Araripe. O teor geral de carbonato nesta fácies, ainda segundo Neumann et al. (2003), geralmente se encontra acima de $65 \%$ e o teor de carbono orgânico abaixo de $4 \%$. Teores muito baixos de pirita, na forma de elementos traços, foram identificados em restos de galhos de plantas superiores parcialmente preservados nesta fácies.

Martill et al. (2008) descreve a ocorrência de estruturas, na forma de chaminés, compostas por dolomitas brechadas ricas em ferro. De acordo com Dana (1969), a ankerita é um carbonato romboédrico, com estrutura análoga à estrutura da dolomita, na qual o ferro ferroso substitui parte do magnésio.

Neste trabalho foram analisadas as propriedades petrofísicas de 64 amostras pertencentes à fácies ritmito da Formação Crato. Tais amostras foram coletadas pela equipe da UFPE e enviadas para o Laboratório de Petrofísica da UFCG, onde foi realizada a preparação final dos plugues e as análises petrofísicas. As propriedades medidas foram: a densidade total e de grãos, porosidade e velocidades elásticas. Todas as análises foram realizadas com as rochas secas e sob temperatura ambiente. Os ensaios de porosidade e densidade foram realizados sob pressão ambiente e as velocidades elásticas foram medidas sob pressões confinantes de 5 até $40 \mathrm{MPa}$.

Utilizando o modelo petrofísico proposto por Soares et al. (2014) e adaptando-o para a determinação de dois componentes minerais, três possíveis cenários foram investigados.

\section{Metodologia}

As amostras de rocha analisadas no Laboratório de Petrofísica da UFCG passam por duas etapas: primeiro a preparação dos plugues, que consiste na serragem e retificação, secagem, pesagem e medição de suas dimensões; e numa segunda etapa, a realização dos ensaios propriamente ditos.

Os ensaios de porosidade, densidade e permeabilidade foram realizados no equipamento Ultraporoperm 500, o qual utiliza a lei de Boyle-Mariotte para medição da porosidade e densidade, e a lei de Darcy para medição da permeabilidade. Este equipamento utiliza gás nitrogênio. As medidas de porosidade e de densidade são realizadas sob pressão e temperatura ambientes. A permeabilidade é medida sob temperatura ambiente e pressão confinante de 500 psi. 
As propriedades elastodinâmicas das rochas foram medidas no equipamento Autolab 500, sob temperatura ambiente e pressões confinantes efetivas entre 5 e 40 $\mathrm{MPa}$. O equipamento mede as velocidades de propagação das ondas $\mathrm{P}$ e $\mathrm{S}$ na direção do eixo dos plugues cilíndricos, sendo que são registradas duas ondas S com polarizações mutuamente perpendiculares (no plano perpendicular ao eixo do plugue).

Para o cálculo da composição mineral a partir das propriedades petrofísicas, fez-se uma adaptação do modelo de Soares et al. (2014). Uma vez que os plugues se encontram secos, a densidade total de um dado plugue pode ser dada por:

$\rho_{B}=\rho_{G}(1-\phi)$

onde $\rho_{G}$ é a densidade de grãos e $\phi$ é a porosidade.

A densidade de grãos é uma média ponderada das densidades dos componentes da matriz. Considerando que a matriz é composta por apenas dois minerais:

$\rho_{\mathrm{G}}=\mathrm{FV}_{\min 1} \rho_{\min 1}+\mathrm{FV}_{\min 2} \rho_{\min 2}$

onde $F V_{\min 1}$ e $F V_{\min 2}$ são as frações volumétricas de calcita e do mineral desconhecido, respectivamente, que compõem as amostras de rocha.

Substituindo a equação (2) na equação (1) e considerando que $\mathrm{FV}_{\min 1}=1-\mathrm{F}_{\mathrm{Vmin} 2}$, temos a equação (3):

$$
F V_{\min }=\frac{\rho_{\mathrm{B}}-\rho_{\min 1}(1-\phi)}{\left(\rho_{\min 2}-\rho_{\min 1}\right)(1-\phi)}
$$

Como se dispõe dos valores de densidade total e porosidade para todas as amostras, através da equação (3), foi possível estimar a composição da matriz mineral dos plugues analisados considerando três cenários: no primeiro cenário as amostras seriam compostas basicamente por calcita e dolomita. No segundo, por calcita e pirita e, por fim, no terceiro cenário, além da calcita haveria a presença de ankerita. No cálculo das frações volumétricas para os três cenários foram adotadas as densidades minerais apresentadas no Quadro 1.

Quadro 1: Densidades adotadas para cálculo das frações volumétricas.

\begin{tabular}{|c|c|}
\hline Mineral & Densidade \\
\hline Calcita & 2,69 \\
\hline Dolomita & 2,84 \\
\hline Pirita & 3,00 \\
\hline Ankerita & 5,00 \\
\hline
\end{tabular}

\section{Resultados}

A Figura 1 apresenta a densidade de grãos medida para as 64 amostras carbonáticas analisadas. Nela observa-se que há três grupos de amostras: o maior grupo de amostras, que exibe densidade de grãos em torno de $2,75 \mathrm{~g} / \mathrm{cm}^{3}$, que é compatível com uma composição majoritariamente calcária, mas não exclusivamente (representado pelos símbolos pretos); um segundo grupo com densidade de grãos próxima de $2,68 \mathrm{~g} / \mathrm{cm}^{3}$, o que sugere a presença de minerais menos densos que a calcita (símbolos azuis); e duas amostras que apresentam densidade de grãos próxima a $2,84 \mathrm{~g} / \mathrm{cm}^{3}$, o que indica a presença expressiva de minerais mais densos que a calcita (símbolos vermelhos).

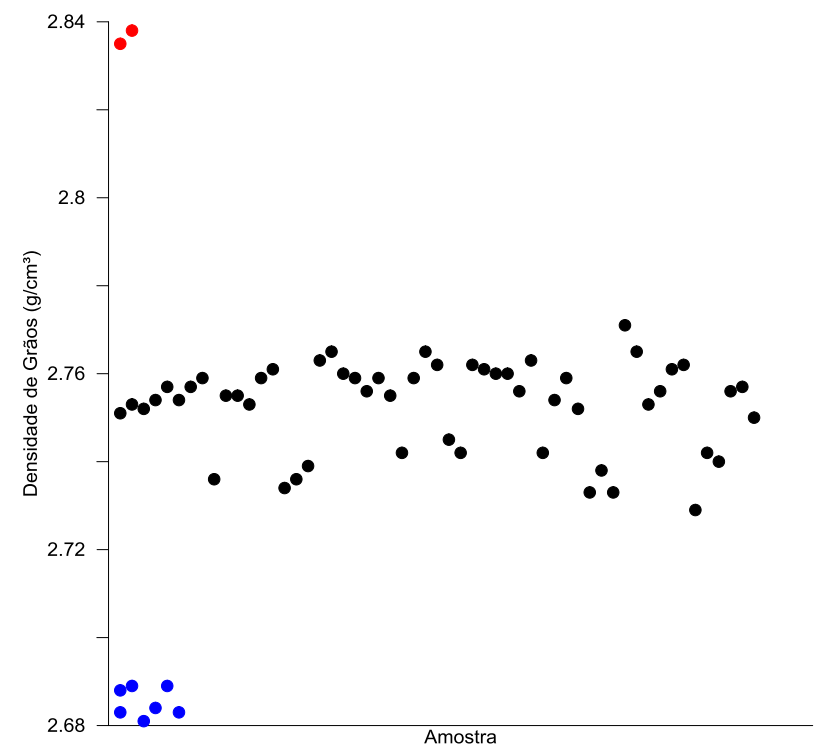

Figura 1: Densidade de grãos das amostras.

A Figura 2 apresenta a relação entre a densidade de grãos e a porosidade das amostras. Os valores de porosidade variam desde aproximadamente $1 \%$ até $18 \%$. A mesma separação das amostras em três grupos é também observada em termos dos valores de porosidade. Há uma relação inversa entre estas duas variáveis, a qual é inesperada. Isto sugere que as amostras de densidade de grãos mais baixas podem conter argilominerais e/ou matéria orgânica, o que reduz a sua densidade e aumenta a porosidade. Já o grupo de maior densidade de grãos, supostamente está afetado por processos diagenéticos (dolomitização/ankeritização) que promoveram a redução dos valores de porosidade e o aumento da densidade média dos grãos.

A Figura 3 apresenta a relação entre a densidade total e a porosidade. Nela observa-se mais uma vez a separação entre os três grupos de amostras, de acordo com a composição mineral. Há uma relação inversa, como esperado, entre as duas variáveis. Na figura são apresentadas as equações das retas de ajuste para os três grupos de amostras.

A Figura 4 apresenta a relação entre a velocidade da onda $\mathrm{P}$ e a porosidade para pressão confinante de 40 MPa. Observa-se, como esperado, uma redução das velocidades com o aumento da porosidade. A dispersão deve ser causada pelo tipo de porosidade predominante.

Wyllie et al. (1958) propuseram um modelo empírico que relaciona a velocidade compressional da rocha $(\mathrm{Vp}) \mathrm{com}$ a porosidade $(\varnothing)$ e as velocidades da matriz (Vma) e do fluido (Vf), conforme a equação (4). 
$\frac{1}{V p}=\frac{\emptyset}{V_{f}}+\frac{1-\emptyset}{V m a}$

A equação de Wyllie é indicada para rochas com predominância de porosidade primária. Rochas com porosidade vugular ou secundária tendem a apresentar velocidades mais altas do que as previstas pela equação de Wyllie.

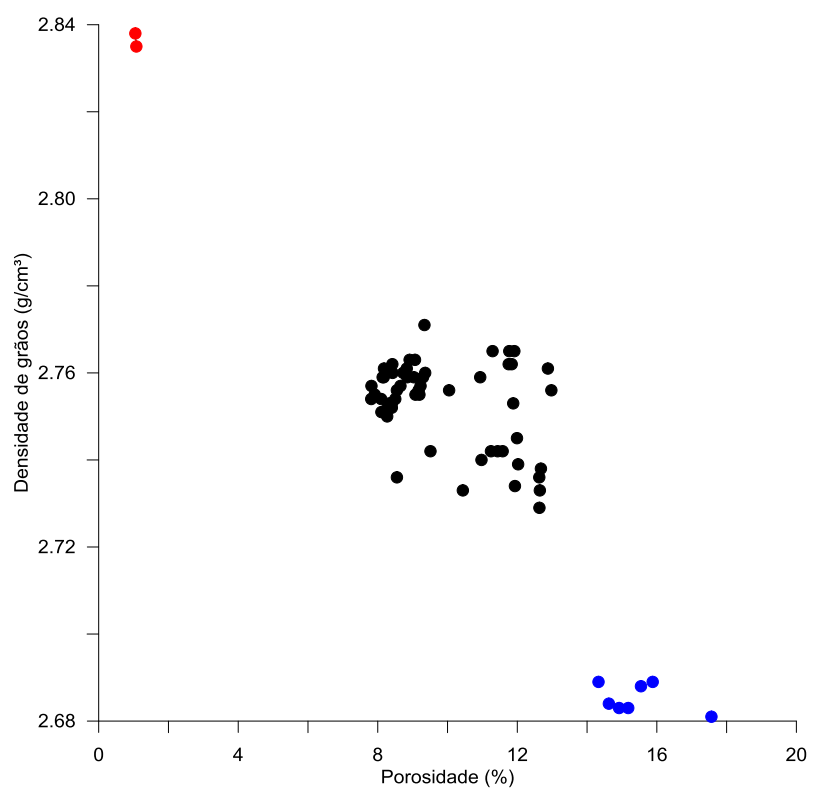

Figura 2: Densidade de grãos versus porosidade.

Raymer et al. (1980) sugeriram uma equação alternativa à de Wyllie, a qual não possui as mesmas limitações quanto ao tipo de porosidade. A equação 5 é a equação de Raymer para rochas com porosidade menor que $37 \%$.

$$
V p=(1-\emptyset)^{2} V_{m a}+\emptyset V_{f}
$$

As amostras analisadas apresentam velocidades da onda $P$ entre as velocidades previstas pelas equações de Raymer e de Wyllie. Isto ocorre porque rochas carbonáticas podem apresentar tanto porosidade primária quanto secundária.

As Figuras 5 e 6 apresentam as velocidades das ondas cisalhantes $S_{1}$ e $S_{2}$, respectivamente, versus a porosidade. O comportamento dos gráficos ocorre como o esperado, ou seja, há uma redução das velocidades com o aumento da porosidade.

A Figura 7 apresenta a razão entre as velocidades cisalhantes. Nota-se que, com exceção de uma amostra, a anisotropia máxima é de cerca de $10 \%$, podendo-se considerar estas amostras como aproximadamente isotrópicas.

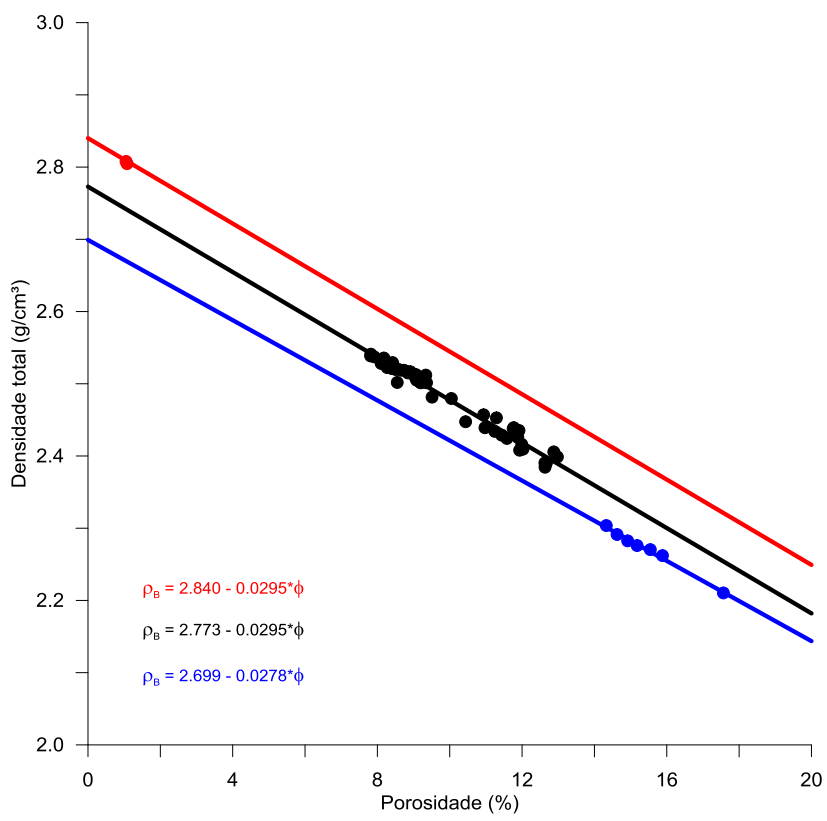

Figura 3: Densidade total versus porosidade.

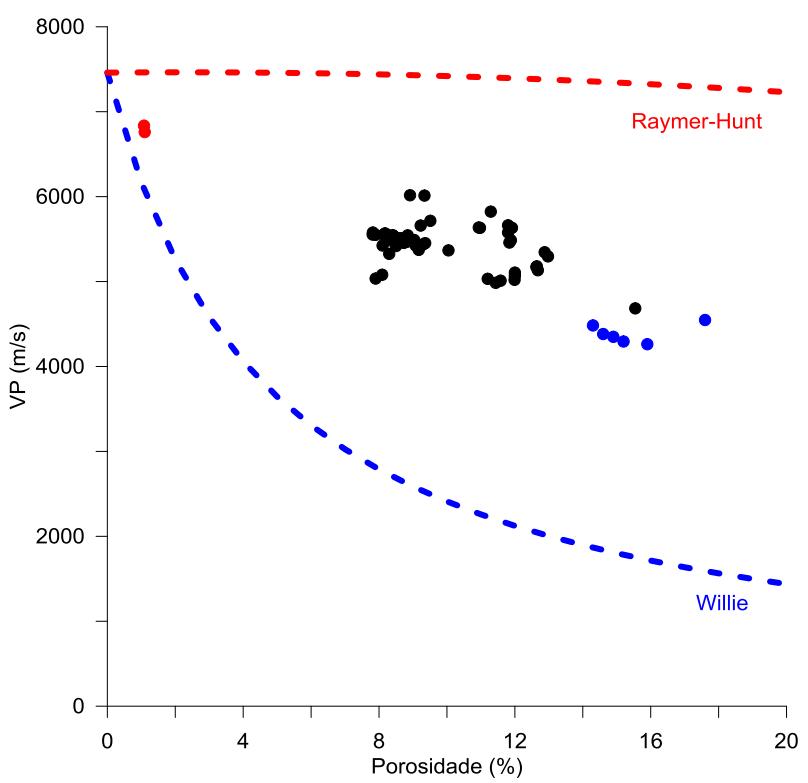

Figura 4: Velocidade da onda $\mathrm{P}$ versus porosidade para pressão confinante de $40 \mathrm{MPa}$.

A Figura 8 apresenta a relação entre a velocidade da onda P a 40 MPa e a fração volumétrica de dolomita, ou seja, considerando o cenário 1 . Nesta figura vê-se que a principal variável que controla os valores de velocidade é a fração volumétrica do mineral mais denso, mas que há um efeito secundário do tipo de porosidade predominante. Este resultado também é válido para os demais cenários.

Considerando o cenário 1 , onde as amostras são compostas por calcita e dolomita, a Figura 9 apresenta as densidades de grãos e as frações volumétricas para 
todas as amostras analisadas. Considerando agora o cenário 2, onde as amostras são compostas por calcita e pirita, a Figura 10 apresenta as mesmas propriedades. Finalmente para o cenário 3 , onde as amostras são compostas por calcita e ankerita, a Figura 11 apresenta as correspondentes densidades de grãos e frações volumétricas.

O cenário 1 é considerado pouco provável porque a literatura não discute a presença massiva de dolomita na composição dos ritmitos (fácies 5 de Neumann et al., 2003), além de análises petrográficas realizadas pela equipe da UFPE não terem detectado presença considerável de dolomita.

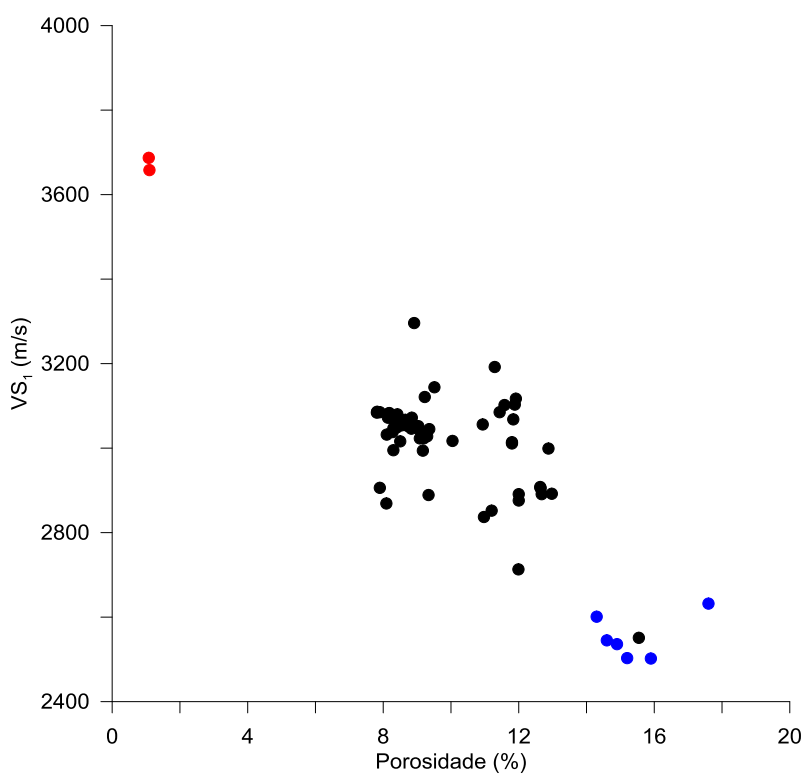

Figura 5: Velocidade da onda $\mathrm{S}_{1}$ versus porosidade para pressão confinante de $40 \mathrm{MPa}$.

O cenário 2 apresenta probabilidade razoável, pois a literatura descreve a ocorrência de pirita apenas como elemento traço, identificada em restos de galhos de plantas parcialmente preservados.

Outro cenário possível é o 3, visto que Martill et al. (2008) discute a presença de dolomita rica em ferro (ankerita) em estruturas colunares presentes em áreas específicas da Bacia do Araripe.

\section{Conclusões}

Neste trabalho foram identificados três grupos de amostras, os quais podem ser individualizados através dos valores de densidade de grãos e de porosidade. Isto ocorre devido à variação na composição mineral da matriz rochosa, como indicado nos três possíveis cenários investigados.

As velocidades elásticas apresentam uma relação inversa com a porosidade. As velocidades elásticas são controladas, principalmente, pela fração volumétrica do mineral mais denso, além de haver o efeito secundário do tipo de porosidade predominante.
Análises adicionais precisam ser realizadas a fim de definir qual o cenário de composição mineral mais realista. De qualquer forma, existem três grupos de amostras que, além da calcita, contém teores variados do segundo mineral, o qual pode ser dolomita, ankerita ou pirita.

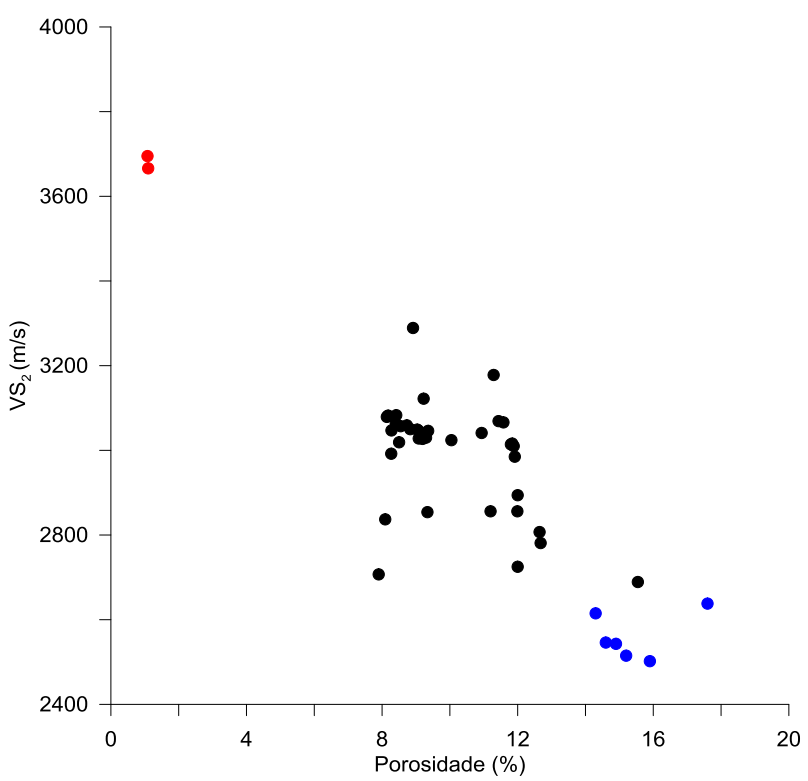

Figura 6: Velocidade da onda $S_{2}$ versus porosidade para pressão confinante de $40 \mathrm{MPa}$.



Figura 7: Razão $\mathrm{VS}_{1} / \mathrm{S}_{2}$ versus porosidade para pressão confinante de $40 \mathrm{MPa}$. 


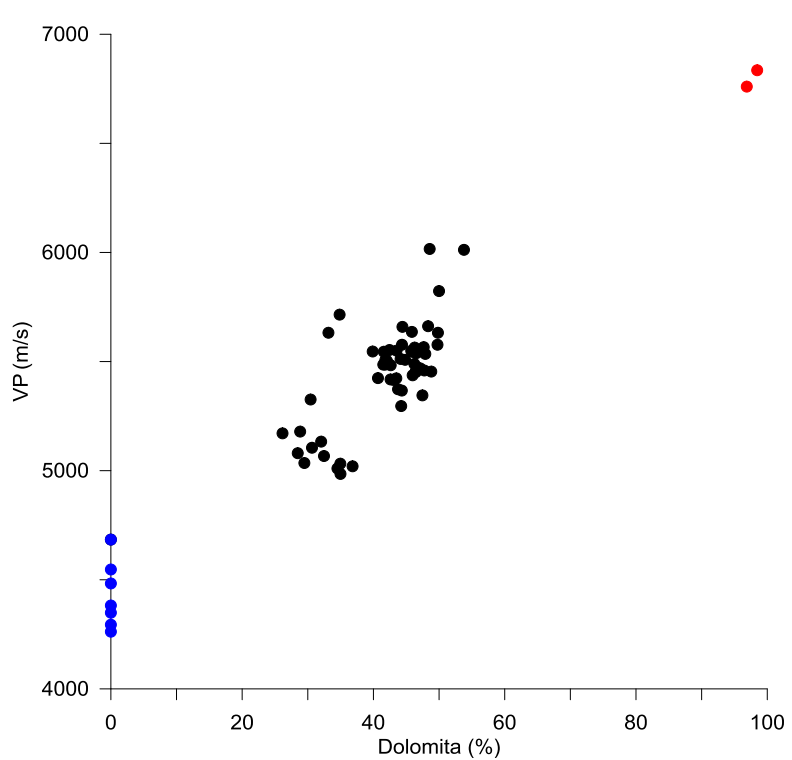

Figura 8: Velocidade da onda $\mathrm{P}$ a $40 \mathrm{MPa}$ versus fração volumétrica de dolomita (cenário 1).

\section{Agradecimentos}

Os autores agradecem aos convênios firmados entre a PETROBRAS e as Universidades Federais de Pernambuco e de Campina Grande, através da Rede Carmod, que permitiram a realização deste trabalho.

\section{Referências}

ASSINE, M. L. Análise estratigráfica da Bacia do Araripe, Nordeste do Brasil. Revista Brasileira de Geociências, 22 (3) : 289-300, setembro de 1992.

CATTO, B. Laminitos microbiais no membro Crato (neoaptiano), Bacia do Araripe, nordeste do Brasil. Dissertação de mestrado. Instituto de Geociências e Ciências Exatas da Universidade Estadual Paulista, Campus de Rio Claro, 2015.

DANA, J.D. Manual de Mineralogia. Livros Técnicos e Científicos Editora S.A. 1969.

MARTILL, D. M.; LOVERIDGE, R. F.; HEIMHOFER, U. Dolomite pipes in the Crato Formation fossil lagerstätte (Lower Cretaceous, Aptian), of northeastern Brazil. Cretaceous Research 29, 2008, pp. 78-86.

NEUMANN, V.H.; BORREGO, A.G.; CABRERA, L.; DINO, R. Organic matter composition and distribution through the Aptian-Albian lacustrine sequences of the Araripe Basin, northeastern Brazil. International Journal of Coal Geology 54, 21- 40, 2003.

ROSA, A. J. ; CARVALHO, R. S.; XAVIER, J.A.D. Engenharia de reservatórios de petróleo. Rio de Janeiro: Interciência, 2006.

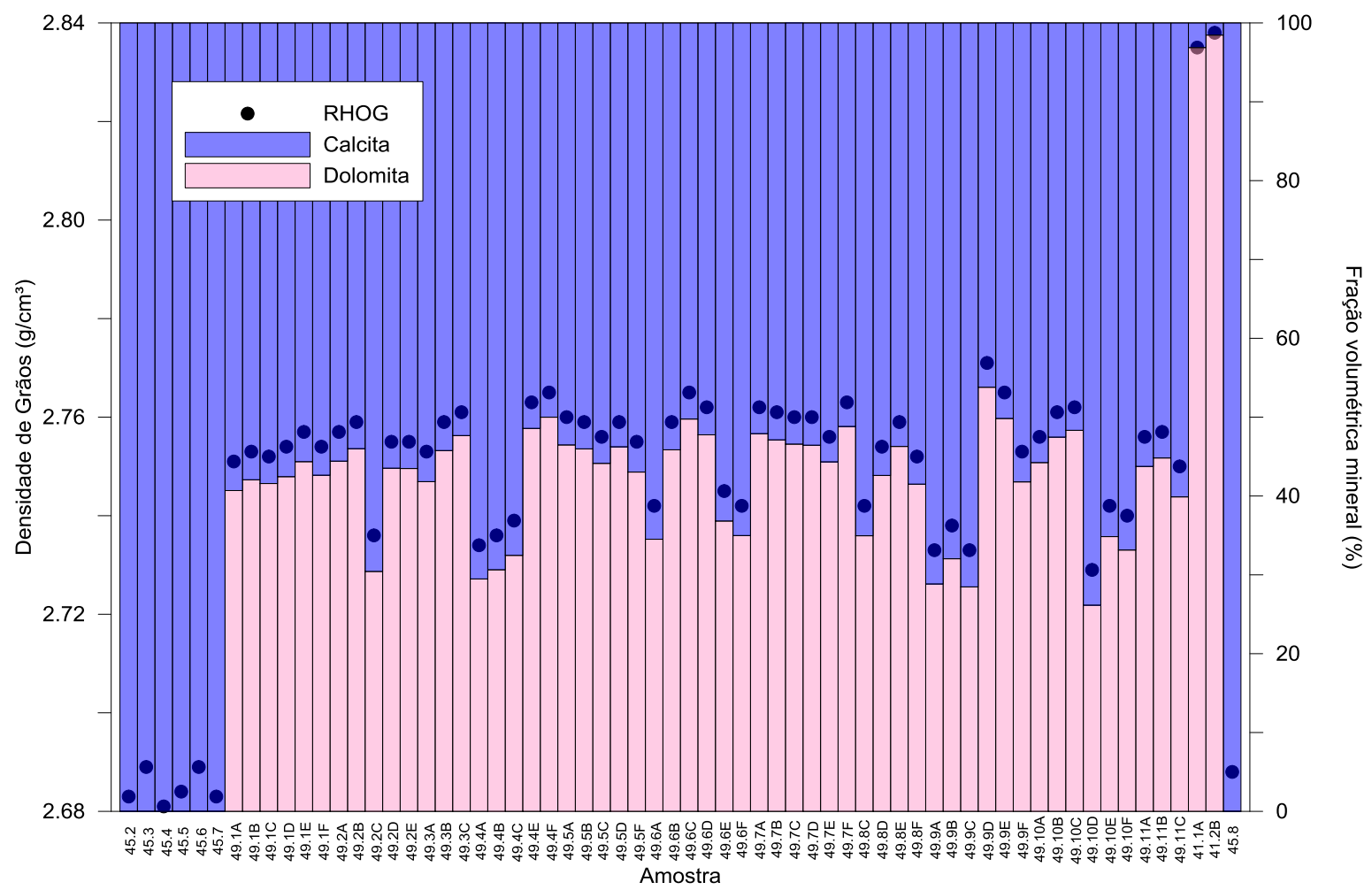

Figura 9: Composição mineral supondo a presença de dolomita. 


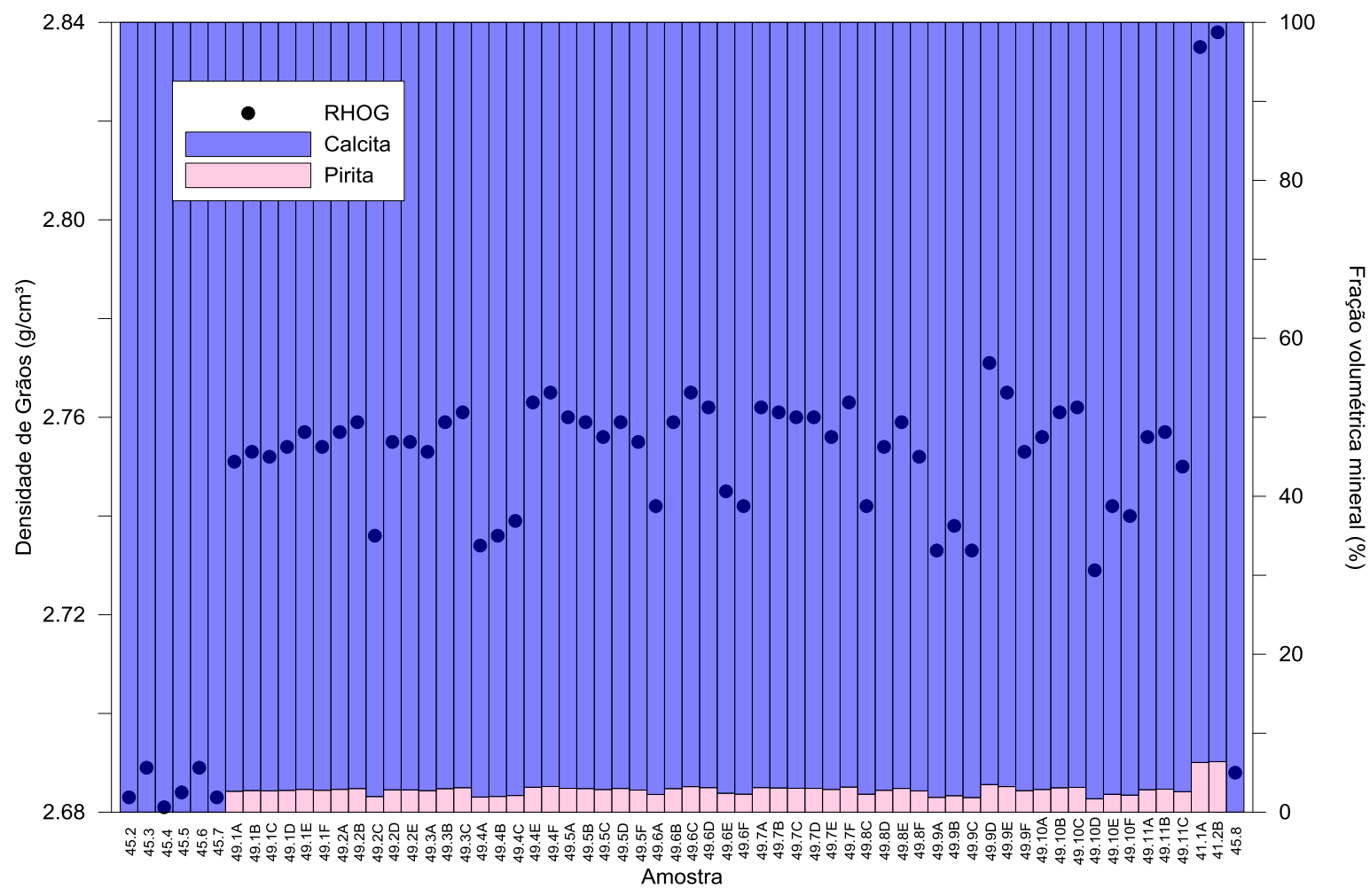

Figura 10: Composição mineral supondo a presença de pirita.



Figura 11: Composição mineral supondo a presença de ankerita. 